\title{
Admixture with indigenous people helps local adaptation: admixture-enabled selection in Polynesians
}

\author{
Mariko Isshiki ${ }^{1}$, Izumi Naka ${ }^{1}$, Ryosuke Kimura², Nao Nishida ${ }^{3}$, Takuro Furusawa ${ }^{4}$, Kazumi Natsuhara ${ }^{5}$, \\ Taro Yamauchi', Minato Nakazawa ${ }^{7}$, Takafumi Ishida ${ }^{1}$, Tsukasa Inaoka ${ }^{8}$, Yasuhiro Matsumura9 \\ Ryutaro Ohtsuka ${ }^{10}$ and Jun Ohashi ${ }^{1 *}$ (])
}

\begin{abstract}
Background: Homo sapiens have experienced admixture many times in the last few thousand years. To examine how admixture affects local adaptation, we investigated genomes of modern Polynesians, who are shaped through admixture between Austronesian-speaking people from Southeast Asia (Asian-related ancestors) and indigenous people in Near Oceania (Papuan-related ancestors).

Methods: In this study local ancestry was estimated across the genome in Polynesians (23 Tongan subjects) to find the candidate regions of admixture-enabled selection contributed by Papuan-related ancestors.

Results: The mean proportion of Papuan-related ancestry across the Polynesian genome was estimated as $24.6 \%$ $(\mathrm{SD}=8.63 \%)$, and two genomic regions, the extended major histocompatibility complex ( $\mathrm{XMHC}$ ) region on chromosome 6 and the ATP-binding cassette transporter sub-family C member 11 (ABCC11) gene on chromosome 16, showed proportions of Papuan-related ancestry more than 5 SD greater than the mean $(>67.8 \%)$. The coalescent simulation under the assumption of selective neutrality suggested that such signals of Papuan-related ancestry enrichment were caused by positive selection after admixture (false discovery rate $=0.045$ ). The $A B C C 11$ harbors a nonsynonymous SNP, rs17822931, which affects apocrine secretory cell function. The approximate Bayesian computation indicated that, in Polynesian ancestors, a strong positive selection $(s=0.0217)$ acted on the ancestral allele of rs17822931 derived from Papuan-related ancestors.
\end{abstract}

Conclusions: Our results suggest that admixture with Papuan-related ancestors contributed to the rapid local adaptation of Polynesian ancestors. Considering frequent admixture events in human evolution history, the acceleration of local adaptation through admixture should be a common event in humans.

Keywords: Positive selection, Admixture-enabled selection, Admixture, Rapid adaptation, Population genomics, Genetic ancestry, Polynesia

\footnotetext{
*Correspondence: juno-tky@umin.ac.jp

${ }^{1}$ Department of Biological Sciences, Graduate School of Science, The University of Tokyo, Tokyo 113-0033, Japan

Full list of author information is available at the end of the article
}

\section{Background}

The human occupation of Oceania began approximately 47,000 years ago [38]. The first immigrants settled Sahul, a continent that comprised the land masses of presentday Australia, New Guinea, and the surrounding small islands. They are considered the ancestors of modern Papuans and Aboriginal Australians. They colonized the 
islands of New Britain and New Ireland, reaching the Solomon Islands by 28,000 years ago [65]. This region of initial colonization is known as Near Oceania. Probably due to the large expanse of ocean to the east of Near Oceania, Remote Oceania, which includes eastern part of the Solomon Islands, Vanuatu, Fiji and all the islands of Polynesia, remained unoccupied until the Late Holocene.

Austronesian (AN)-speaking people from Southeast Asia who possessed the advanced navigation skills necessary for a long-distance voyage first colonized Remote Oceania. They are called the Lapita people after their culture, Lapita, which is characterized by pottery decorated with distinctive motifs. Remains of their characteristic pottery suggest that they originated in Taiwan and arrived in the Bismarck Archipelago about 3500 years ago $[6,28,57]$. Lapita people then expanded into Remote Oceania using their advanced navigation skills. They reached western Polynesia, Tonga and Samoa, 2900-2700 years ago $[9,52,53]$, and finally Hawaii, Easter Island and New Zealand by $1200-800$ years ago [4, 17, 19]. They are considered the direct ancestors of modern Polynesians.

Several genetic studies have found that about $20-30 \%$ of the modern Polynesian genome was derived from Papuan-related ancestry, and the rest was derived from Asian-related ancestry [24, 27, 56, 66], indicating that the Asian-related Polynesian ancestors admixed with indigenous people in Near Oceania, the Papuan-related ancestors, during their expansion from Near Oceania to Polynesia. The admixture between Asian- and Papuanrelated ancestors has been estimated to have occurred about 3000 years ago $[47,66]$.

Homo sapiens populations have experienced admixture many times during the species' expansion and adaptation all over the world [16]. Admixture with populations from different genetic backgrounds would lead to introduction of adaptive genetic variants at intermediate frequencies in the gene pool, and thus can enable rapid adaptation to the local environments. Such admixturemediated adaptation must have played an important role in human evolution. In the case of Polynesians, their Papuan-related ancestors, who had inhabited Oceania for tens of thousands of years, might already have had some genetic components adapted to Oceanian environment at the time of admixture. Therefore, it is expected that if the Polynesian ancestors had acquired genomic regions adaptive to the Oceanian environment through admixture, the frequency of those regions would increase by natural selection, and the regions would contribute to the adaptation of Polynesians. The phenomenon whereby genome regions introduced through admixture increase the fitness of admixed populations is called "adaptive introgression". This phenomenon is well-studied in the case of the admixture between modern humans and archaic hominins, such as Neanderthals and Denisovans [50], and adaptive introgression between modern human populations, often called as admixture-enabled selection, has also been studied in a few specific groups $[7,8,12,15$, $21,22,37,42,44,54,60,68]$. Recently African ancestry enrichment around the human leukocyte antigen $(H L A)$ region and signals of polygenic selection on immune function were observed in Latin American populations, suggesting admixture drove rapid adaptive evolution in human populations [37]. Investigating the effect of admixture on local adaptation in Polynesian genomes is therefore important for deepening our understanding of human evolution.

Previously we detected the signatures of local selective sweeps in Polynesian genomes by comparing the haplotype variation between Tongans and reference populations [27]. In this study, local ancestry was estimated across the genome in the same Polynesian subjects to reveal the effect of admixture on their local adaptation. Genomic regions with particularly high levels of Papuan-related ancestry in Polynesian genomes could have undergone admixture-enabled selection, based on the principle that the proportions of local ancestry are expected to be similar across the genome, unless affected by natural selection. We detected the signatures of admixture-enabled selection from Papuan-related ancestors in two genomic regions (chromosomes 6 and 16) in Polynesians. One of the regions harbored the ATPbinding cassette transporter sub-family $C$ member 11 (ABCC11), and the $A B C C 11$ allele (rs17822931-C) which determines wet earwax, was likely to have experienced a strong positive selection in Polynesians after the admixture. Our results suggest that the admixture with Papuan-related ancestors contributed to the rapid adaptation of Polynesian ancestors to the environment of Oceania.

\section{Results \\ Clustering analysis and Admixture proportion}

Principal component analysis (PCA) and ADMIXTURE analysis [2] were performed on 179 individuals from five Oceanian and three Asian populations (Fig. 1). Percentages of variance was $7.18 \%$ for $\mathrm{PC} 1$ and $3.40 \%$ for $\mathrm{PC} 2$ (Fig. 1b). AN-speaking admixed populations (i.e. Munda, Rawaki and Tongans) were plotted between Papuans (i.e. Gidra) and Asians (i.e. CHB, Ami and Atayal) as expected from their population histories. Two Tongan populations obtained from different studies were clustered. Figure 1c illustrates individual ancestry proportion inferred by ADMIXTURE analysis for numbers of postulated ancestral populations $(\mathrm{K})$ ranging from two to six. $K=5$ 

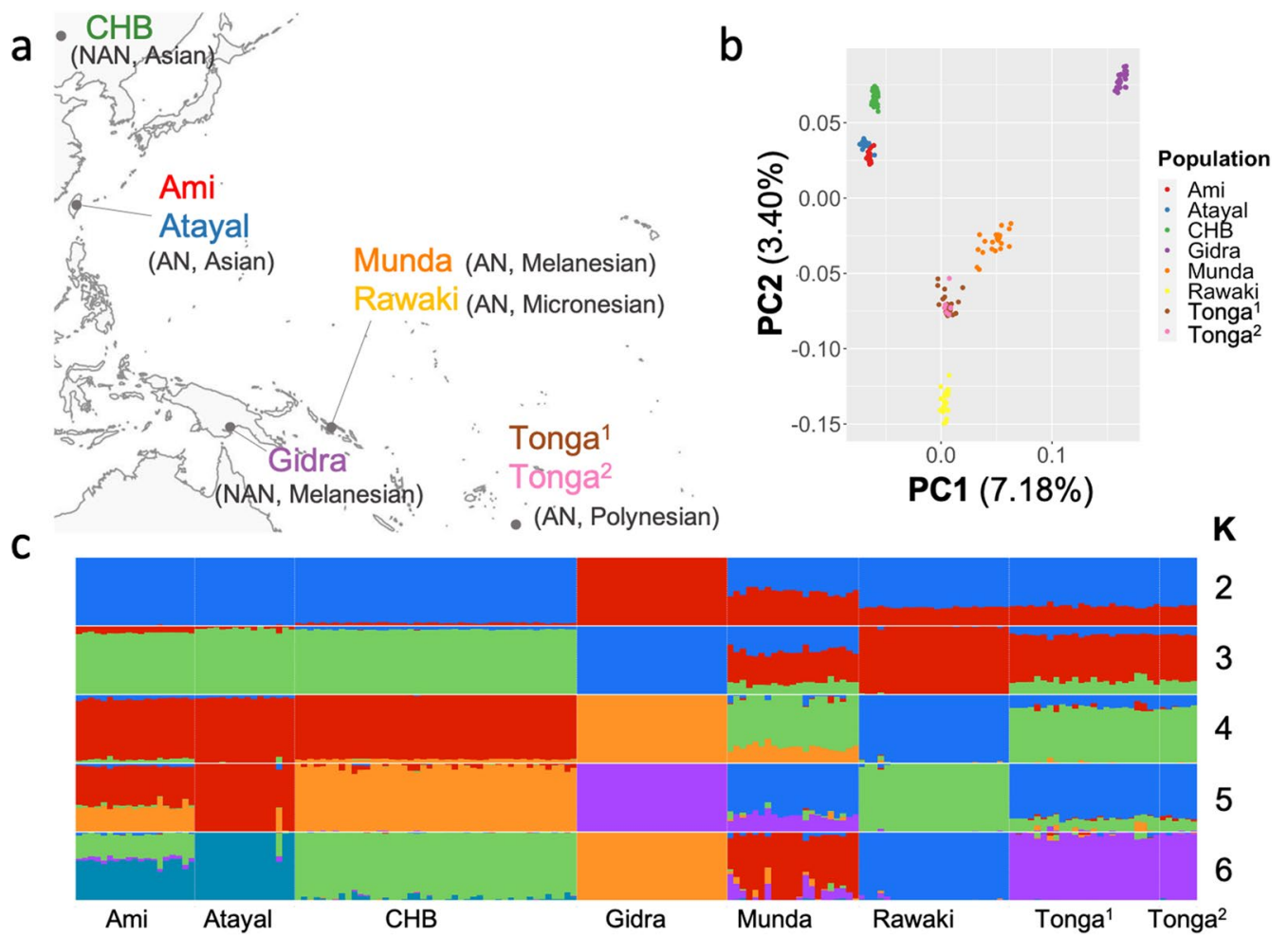

Fig. 1 PCA and ADMIXTURE analysis of eight populations. a A map of eight populations analyzed in this study. AN: AN-speaking population. NAN: NAN-speaking population. b PCA plot for the eight populations. $\mathbf{c}$ Results of ADMIXTURE analysis for K ranging from 2 to 6 . The lowest cross-validation error was obtained for $\mathrm{K}=5$. The map depicted in Fig. 1 (a) was taken from FREEWORLDMAP.NET (https://www.freeworldmaps.net/). 1 Tongans obtained from Kimura et al. [27]. 2Tongans obtained from Qin and Stoneking [48] and Pugach et al. [46]

provided the lowest cross-validation error (Additional file 1: Fig. S1). Assuming red and blue components for $\mathrm{K}=2$ as Asian- and Papuan-related ancestries, respectively, the proportion of Asian-related ancestry for Tongans was estimated as $71.4 \%(\mathrm{SD}=2.21 \%)$.

The f3 statistics for Tongans were estimated using the Gidra as a proxy for Papuan-related ancestors and the Han Chinese population of Beijing (CHB) [61] or Aboriginal Taiwanese [30, 46, 48] for Asian-related ancestors, to examine whether they descended from a mixture of the two ancestral populations. Concordant with previous studies, negative f3 statistics were observed regardless of which populations were assumed as a proxy for Asian-related ancestors, indicating that Tongans or Polynesians were the descendants of a mixture of Papuan- and Asian-related ancestors (Table 1 ).

The proportion of Asian-related ancestry in Polynesian genomes was estimated using the f4 ratio test, assuming the phylogeny shown in Additional file 1: Figure S2. The proportion of Asian-related ancestry was estimated as $67.4 \%(\mathrm{SE}=1.22 \%, \mathrm{Z}=55.2)$.
Table 1 Results of 3-Population test for Tongans

\begin{tabular}{llllll}
\hline $\begin{array}{l}\text { Papuan- } \\
\text { related } \\
\text { ancestors }\end{array}$ & $\begin{array}{l}\text { Asian- } \\
\text { related } \\
\text { ancestors }\end{array}$ & f3 & SE & Z & SNPs \\
\hline Gidra & Taiwan & -0.0174 & 0.000737 & -23.7 & 49523 \\
Gidra & CHB & -0.0108 & 0.000753 & -14.3 & 49523 \\
\hline
\end{tabular}

$\mathrm{SE}$, standard error

\section{Natural selection acted on the genomic regions derived from Papuan-related ancestors in Polynesians}

The contributions of Asian- and Papuan-related ancestry across the Polynesian genome (Papuan versus Asian ancestry) were measured using the Effective Local Ancestry Inference (ELAI) algorithm [15] with CHB and Gidra as proxies for Asian- and Papuan-related ancestors, respectively. Figure 2 shows the mean proportion of Papuan-related ancestry across the Polynesian genome, estimated by the ELAI program with 100 admixture generations. The mean proportion of Papuanrelated ancestry was estimated as $24.6 \%(\mathrm{SD}=8.63 \%)$, which corresponded with the previous studies $[24,66]$. 


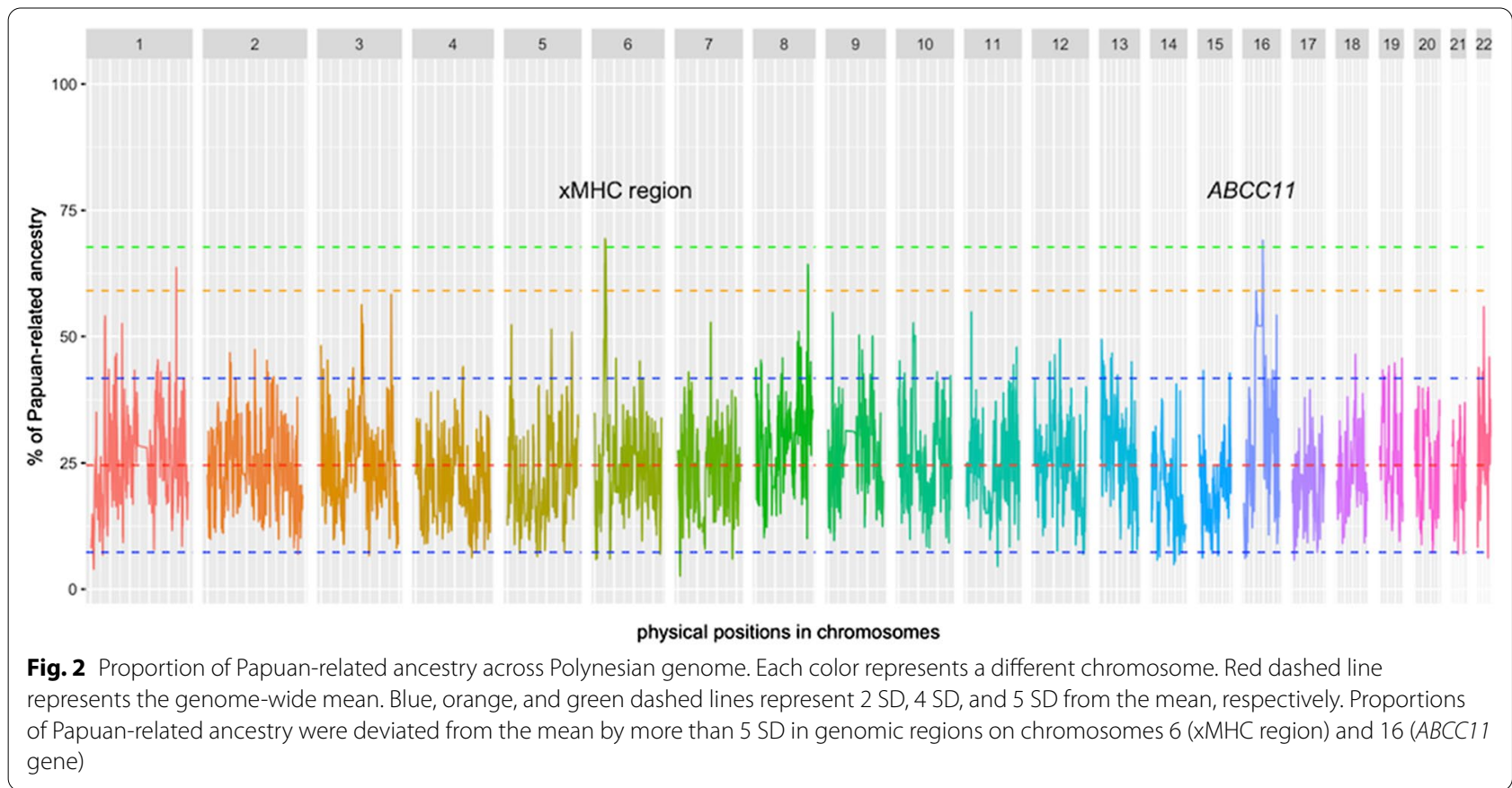

Two genomic regions displayed proportions of Papuanrelated ancestry more than $5 \mathrm{SD}$ greater than the mean (Fig. 2) (chr6:26471596-28011652 and chr16:4822647948266831). The detected regions on chromosome 6 and 16 contained 51 SNPs and 4 SNPs, respectively. The high Papuan-related ancestry region on chromosome 6 (Papuan-related ancestry proportion: 67.72-69.37\%) was located within the extended major histocompatibility complex (xMHC) region. 43 genes such as histone protein genes and DNA-binding protein genes are clustered within the region. Since this region contained 51 SNPs in strong LD, the candidate genes cannot be detected from the current dataset. The high Papuan-related ancestry region on chromosome 16 (Papuan-related ancestry proportion: $67.85-69.03 \%)$ contained only the $A B C C 11$ gene.

Since local ancestry inference results can be very sensitive to the choice of source populations, we performed ELAI analysis assuming Aboriginal Taiwanese, who are considered to be closer to Asian-related ancestors of Polynesians than CHB are, as a proxy for Asian-related ancestors. The two genomic regions were also detected in the ELAI analysis (Additional file 1: Fig. S3), suggesting that the high Papuan-related ancestry regions did not come from the differentiation between $\mathrm{CHB}$ and Asianrelated ancestors of Polynesians.

A recent study demonstrated that Polynesian genomes contained European and Native American ancestries [20]. Native American ancestry was observed in Eastern Polynesians while European ancestry was observed in all Polynesian populations analyzed in the study. Thus, we conducted ELAI analysis with three-way admixture model assuming CEU from 1000 Genomes Project Phase 3 [1] as a proxy for European-related ancestors. Although a relatively small contribution from European-related ancestors was observed, the highest degrees of Papuanrelated ancestry were also detected in the two genomic regions detected above (Additional file 1: Fig. S4).

\section{Coalescent simulations}

To examine whether genetic drift alone could cause genomic regions to display proportions of Papuanrelated ancestry more than 5 SD greater than the mean, ELAI analysis was conducted for the whole-genome data generated by coalescent-based simulations, assuming selective neutrality (see Methods for details). As shown in Additional file 1: Fig. S5, the mean and SD of Papuanrelated ancestry estimated from the simulation data were similar to those of real data. To evaluate the false discovery rate (FDR) of our approach, we performed 100 independent runs of coalescent-based simulation and subsequent ELAI analysis, and then counted the number of independent genomic regions showing Papuan-related ancestry more than $5 \mathrm{SD}$ above the mean for each simulation run. Out of the 100 simulation runs, no genetic regions showed a proportion of Papuan-related ancestry more than $5 \mathrm{SD}$ above the mean in 91 runs, and the excess was detected in a single genomic region in each of the remaining 9 runs. The threshold (mean +5 sd) used in this study therefore seemed to yield a family-wise 
error rate (FWER) of 0.09 . Under the condition of FWER $=0.09$, our results corresponded to FDR of 0.045 $(=0.09 / 2)$, since two regions showed such deviations in the real genotype data. Thus, the xMHC and $A B C C 11$ regions could be shaped by genetic drift, but are more likely to have been shaped by positive selection since the admixture of the Papuan- and Asian-related ancestors of modern Polynesians.

\section{Selection acted on the earwax-associated SNP (rs17822931) on ABCC11}

To evaluate the intensity of positive selection on the $A B C C 11$ gene, we focused on a nonsynonymous SNP (G180R), rs17822931. This SNP is known to affect apocrine secretory cell function and determine phenotypes such as earwax type and body odor [34,67]. The derived allele of rs17822931, rs17822931-T or 180R, associated with a dry type of earwax and a reduction in body odor, was frequently observed in Northeast and East Asia [34, 67]. The allele frequencies in four Oceanian populations and 40 individuals of HapMap CHB are shown in Fig. 3 and Table 2. The ancestral allele, rs17822931-C or 180G, was found to be dominant in Oceanian populations.

The approximate Bayesian computation with a forward-time simulation was conducted to estimate the selection coefficient, $s$, for rs17822931-C in Tongans.
Here, only simulation runs that resembled the observed allele frequency in Tonga when the run was terminated were accepted. The distribution of $s$ in 10,000 accepted runs is shown in Additional file 1: Fig. S6. The mean of $s$ was 0.0217 , and the $95 \%$ credible interval was 0.0124-0.0309.

\section{Origin of rs 17822931-C in Polynesians}

Next, to confirm if the rs17822931-C allele in Tongans or Polynesians originated from Papuan-related ancestors, rs12445647 was genotyped as a tag SNP for rs17822931$\mathrm{C}$ derived from Papuan-related ancestors. As shown in Table 2, rs12445647-T was observed at a high frequency in Oceanian populations compared to the other populations. More importantly, the $r^{2}$ value, a measure of linkage disequilibrium (LD), between rs12445647-T and rs17822931-C was higher in Polynesian and Papuan populations than those in Asian populations. Such higher LD shared with modern Polynesian and modern Papuan populations implies that haplotype harboring rs12445647-T and rs17822931-C in Polynesians originated mainly from Papuan-related ancestors.

\section{Haplotype and expression level of $A B C C 11$}

The rapid increase in allele frequency of rs17822931-C in Polynesian ancestors might be due to the fact that the

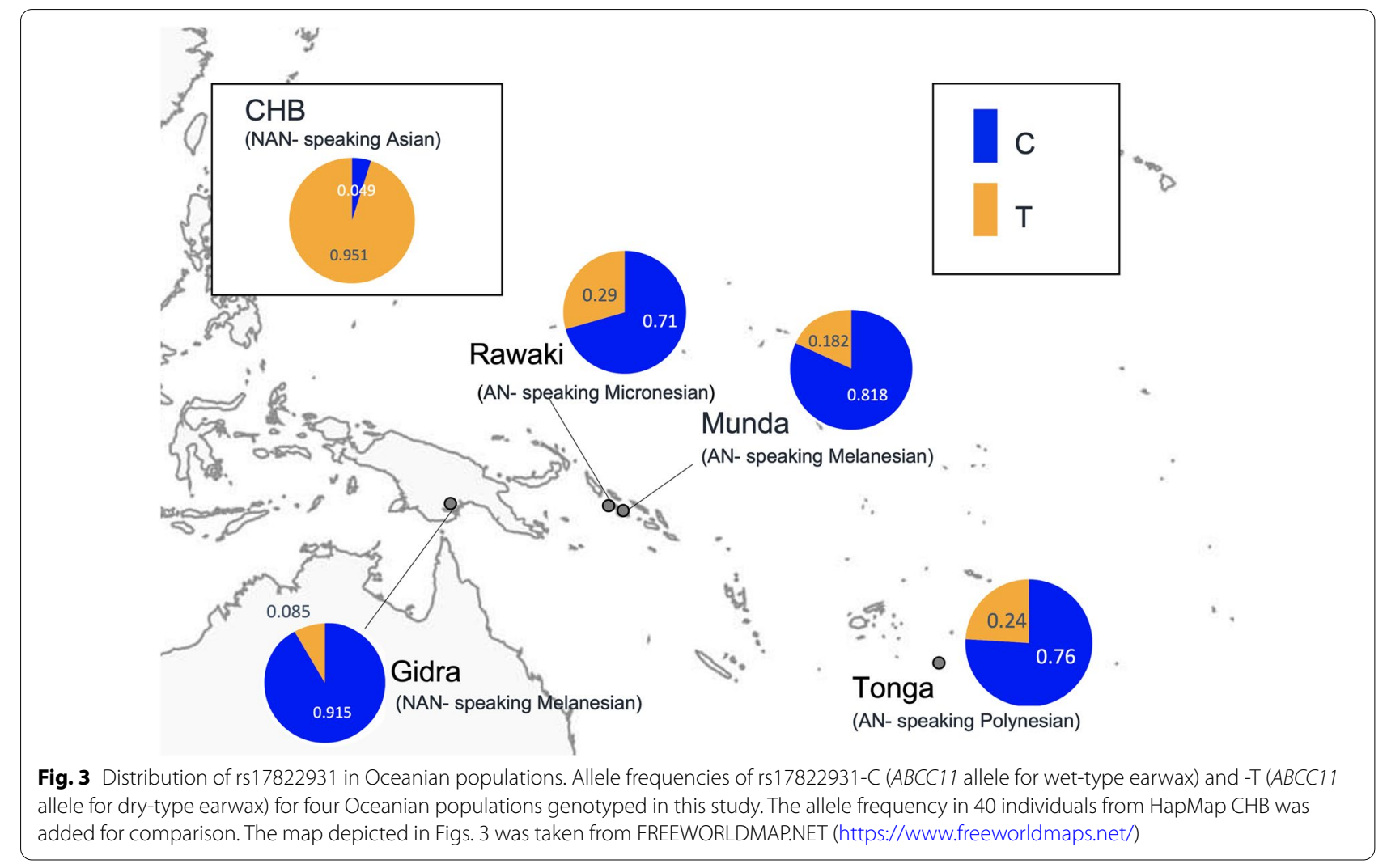


Table 2 Frequency and LD statistics of rs12445647-T and rs17822931-C

\begin{tabular}{|c|c|c|c|c|c|c|}
\hline \multirow[t]{2}{*}{ Populations } & \multirow[t]{2}{*}{ Region } & \multirow[t]{2}{*}{$n$} & \multicolumn{2}{|l|}{ Frequency } & \multirow[t]{2}{*}{$\mathrm{D}^{\prime}$} & \multirow[t]{2}{*}{$\mathrm{R}^{2}$} \\
\hline & & & rs12445647-T & rs17822931-C & & \\
\hline Tonga (Polynesian) & Oceania & 174 & 0.60 & 0.76 & 0.98 & 0.44 \\
\hline Munda (Melanesian) & Oceania & 170 & 0.68 & 0.82 & 1 & 0.47 \\
\hline Rawaki (Micronesian) & Oceania & 107 & 0.62 & 0.71 & 1 & 0.67 \\
\hline Gidra (Papuan) & Oceania & 165 & 0.80 & 0.92 & 0.95 & 0.34 \\
\hline$Y R l^{\mathrm{a}}$ & Africa & 216 & 0.07 & 1 & NA & NA \\
\hline$C E U^{a}$ & Europe & 198 & 0.08 & 0.87 & 1 & 0.01 \\
\hline $\mathrm{CHB}^{\mathrm{b}}$ & Asia & 40 & - & 0.05 & - & - \\
\hline $\mathrm{CHB}^{\mathrm{a}}$ & Asia & 206 & 0.01 & 0.03 & 1 & 0.16 \\
\hline $\mathrm{JPT}^{\mathrm{a}}$ & Asia & 208 & 0 & 0.12 & NA & NA \\
\hline $\mathrm{CHS}^{\mathrm{a}}$ & Asia & 210 & 0.01 & 0.16 & 1 & 0.05 \\
\hline $\mathrm{CDX}^{\mathrm{a}}$ & Asia & 186 & 0.08 & 0.46 & 1 & 0.1 \\
\hline $\mathrm{KHV}^{\mathrm{a}}$ & Asia & 198 & 0.09 & 0.36 & 1 & 0.16 \\
\hline
\end{tabular}

${ }^{a}$ Individuals from 1000 Genomes project Phase 3

${ }^{\mathrm{b}} 40$ individuals from HapMap project used in ELAl analysis

haplotype harboring rs12445647-T and rs17822931-C, which is thought to have been introgressed from Papuanrelated ancestors to the Polynesian ancestors, is functionally different from the haplotype harboring rs12445647-G and rs17822931-C. Since the amino acid in position 180 of the $\mathrm{ABCC} 11$ protein is glycine in both haplotypes, there seems to be no difference in the protein function. We therefore examined the possibility that the $A B C C 11$ mRNA expression level was different between the haplotype harboring rs12445647-T and rs17822931-C and the haplotype harboring rs12445647-G and rs17822931-C, using publicly available data: genotype data of the 1000 Genomes Project Phase 3 populations [1] and microarray data of the HapMap3 populations [13, 18, 59] obtained from the ArrayExpress database at EMBL-EBI. Since no significant association of rs12445647 with the $A B C C 11$ expression level was observed in 217 unrelated subjects with the rs17822931-CC genotype (Additional file 1: Fig. S7), haplotype harboring rs12445647-T and rs17822931$\mathrm{C}$, originated from Papuan-related ancestors, does not seem to be a special haplotype that affects the expression level of the $A B C C 11$ gene.

\section{Discussion}

The results of PCA and ADMIXTURE were consistent with previous studies, suggesting that most fraction of Polynesian genomes were derived from Asian-related ancestry [24, 27, 46, 56, 66]. The f3 statistics revealed that Polynesians experienced admixture between Papuan- and Asian-related ancestors (Table 1). The proportion of Asian-related ancestry was estimated as $71.4 \%$ and $67.4 \%$ by ADMIXTURE $(K=2)$ and the $f 4$ ratio test, respectively. The mean proportion of Asian-related ancestry across the Polynesian genome was estimated to be $75.4 \%(\mathrm{SD}=8.63 \%)$ in ELAI analysis. The estimates were equivalent to the proportions estimated by STRU CTURE analysis [45] in our previous study [27]. Based on these results, the proportion of Asian-related ancestry in the Polynesian genome is expected to fall within $70-80 \%$.

Two genomic regions, $\mathrm{xMHC}$ and $A B C C 11$, with proportions of Papuan-related ancestry greater than $5 \mathrm{SD}$ above the mean were detected in this study (Fig. 2). To examine the possibility that the excess of Papuanrelated ancestry was an artifact of the reference populations assumed, we used other reference populations to confirm the results. The genomic regions of $\mathrm{xMHC}$ and $A B C C 11$ were detected even when Aboriginal Taiwanese were used as a proxy for Asian-related ancestry and when the recent contact of Europeans was assumed (Additional file 1: Figs. S3 and S4). Thus, it is plausible that the detected regions were not an artifact of the reference populations assumed. However, we cannot deny the possibility that the excess of Papuan-related ancestry came from the differentiation between the Lapita and the current East Asian populations as the Lapita people, the direct Asian-related ancestors of Polynesians, were distinct from extant East Asian populations today [56]. A coalescent simulation assuming selective neutrality suggested that these regions were likely to have been subjected to admixture-enabled selection (i.e., The admixture with Papuan-related ancestors have contributed to the rapid local adaptation of Polynesian ancestors). One of the two candidate regions of admixture-enabled selection was located in the $\mathrm{xMHC}$ region. This region overlapped the candidate regions for selective sweeps identified in our previous study with the same genotype data of 
Tongans [27]. Genes involved with various biological processes such as the immune response and epigenetic regulation of gene expression clustered within the region. However, a single candidate polymorphism subjected to positive selection was difficult to be identified from many SNPs that exist in the region due to strong LD between them. Thus, in this study, we focused on the other region, where only the $A B C C 11$ gene is located as a protein-coding gene. This region was not detected in our previous scan for positive selection using LD-based methods [27]. A number of LD-based methods, such as REHH [55], iHS [63], rMHH and rHH [26], have been developed to detect signatures of recent positive selection acting on beneficial derived allele. In the LD-based methods, the ratio of the degree of LD (e.g., extended haplotype homozygosity in $\mathrm{REHH}$ ) between the derived allele and the ancestral allele is expressed as a test statistic. The test statistic for beneficial derived allele is expected to be larger than that for neutral derived allele at the same frequency, since the former exhibits higher degree of LD. LD-based methods are suited for detecting recent positive selection acting on derived allele, but would not show high statistical power for that on ancestral allele in an admixed population, since haplotypes harboring the ancestral allele have already had many recombination events before the admixture in ancestral populations and no extended LD is observed even if the ancestral allele rapidly increases its frequency after the admixture. This is thought to be the main reason that the positive selection that acted on the $A B C C 11$ gene was not detected in our previous study [27].

A nonsynonymous SNP of $A B C C 11$, rs17822931, which affects apocrine secretory cell function and determines the type of earwax and body odor an individual produces, exhibited a large difference in frequency between East Asians and Oceanian populations (Fig. 3). Analysis of the tag SNP, rs12445647, suggested that the majority of rs17822931-C alleles in Polynesians originated mainly from Papuan-related ancestors, not from Asian-related ancestors (Table 2). Therefore, the frequency of rs17822931-C had been low in Polynesian ancestors at the time of admixture and has been rapidly increased due to positive selection since then. The rs17822931-T, a derived allele of rs17822931, has been shown to have experienced positive selection in East Asians as an adaptation to a colder climate, with a selection coefficient estimated as approximately 0.01 $[26,39]$. The selection coefficient of rs17822931-C was estimated to be 0.0217 in Polynesians (Additional file 1: Fig. S6), indicating that the positive selection acting on the ancestral allele in Polynesian ancestors was stronger than that on the derived allele in East Asians. To the best of our knowledge, this study is the first report of positive selection having acted on rs17822931-C, an ancestral allele associated with the production of wet earwax.

The frequency of rs17822931-T shows the northsouth gradient in East Asia [67]. Considering that rs17822931-T experienced positive selection in northeast Asia [39], it is likely that the observed frequency gradient along the latitude may be a result of the shifting balance of which allele was selected more strongly in each environment (e.g. climatic conditions, temperature and pathogen prevalence). However, the evolutionary significance of rs17822931 is still unknown. One possible explanation for positive selection acting on rs17822931-C in Polynesian ancestors is the association of rs17822931 with the amount of apocrine colostrum secretion [36]. Colostrum has an important role in the development of the immune system in newborns [62]. Women with the rs17822931-C allele are significantly less likely to lack colostrum and can produce significantly more colostrum than women without rs17822931-C [36]. Since various pathogens were present in tropical region, colostrum may have been important until the development of modern medical technologies. Genes involved in immune function often present strong signatures of selection and admixtureenabled selection against immune-related genes has been reported in admixed populations $[7,8,12,15,21$, $22,42,44,49,54,60,68]$. Consistently, as $\mathrm{xMHC}$ region also contains genes involved in immune responses against pathogens, infectious disease is a possible driver of the selection in these regions. However, as well as the high apocrine secretion, the $\mathrm{ABCC} 11$ wild-type protein has a function to transport various substrates such as bile acids, conjugated steroids, and cyclic nucleotides [11]. Since these substrates were involved with various physiological processes, further investigation is necessary to clarify the driving force of the positive selection acted on the $A B C C 11$ in Polynesian ancestors.

Two recent ancient DNA studies suggested that the first immigrants into Remote Oceania almost entirely lacked Papuan-related ancestry components [31, 56]. Thus, it is possible that admixture occurred after the settlement of Polynesian ancestors in Remote Oceania dissimilar to the postulated population history in this study that Polynesian ancestors got admixed with Papuan-related ancestors before the expansion into Remote Oceania. Even though the location and timing of the admixture event may need further investigation, there is no doubt that admixture-enabled selection has occurred in Polynesian ancestors after the admixture with Papuan-related ancestors. 


\section{Conclusions}

In this study, we detected two genomic regions subjected to admixture-enabled selection in Polynesians. It is considered that de novo mutations adaptive to the environment generally takes much longer time to reach high frequencies. Therefore, for Asian-related ancestors of Polynesians, it would have been advantageous to acquire pre-existing genetic materials through admixture with Papuan-related ancestors who had already adapted to over tens of thousands of years. The acceleration of adaptation is also observed in Latin American populations [37]. As Homo sapiens have experienced admixture many times in the last few thousand years [16], admixture-enabled selection should be a common event in humans.

\section{Methods}

\section{Subjects and data}

A genome-wide SNP dataset comprising 24 Tongan individuals, AN-speaking Polynesians living in Ha'apai Island and Nuku'alofa of the Kingdom of Tonga, and 24 individuals from Gidra, NAN-speaking Melanesians (Papuans) in the lowlands of Western Province, Papua, New Guinea [27], 21 individuals from Munda, AN-speaking Melanesians in the New Georgia Islands in the western part of the Solomon Islands [21], 24 individuals from Rawaki, ANspeaking Micronesians migrated from the overpopulated Gilbert Islands (Kiribati) to the New Georgia Islands in the 1960s. All individuals were genotyped using Affymetrix GeneChip ${ }^{\circledR}$ Human Mapping 250 K Nsp SNP array. After merged with the HapMap genotype data of 45 unrelated individuals from CHB [61], the dataset consisted of 231,049 autosomal SNPs.

Since Lapita people, the direct ancestors of Polynesian people, are suspected to have originated in Taiwan, the other dataset which includes Aboriginal Taiwanese was also prepared. 35 Aboriginal Taiwanese (16 individuals from Atayal and 19 individuals from Ami) and six Tongans $[30,46,48]$ were added to the above dataset. After SNPs with a genotyping rate lower than 0.95 were filtered out, 179 individuals and 49,523 SNPs were left.

\section{PCA and ADMIXTURE}

A principal component analysis (PCA) and ADMIXTURE analysis were performed on the second dataset comprising of 179 individuals and 49,523 autosomal SNPs. PCA was conducted with using PLINK software v1.90b5.2 [10]. ADMIXTURE analysis was carried out using ADMIXTURE version 1.3.0 [2] for different values of $K$ (from $K=2$ through $K=6$ ). Cross-validation procedure implemented in ADMIXTURE package was performed to find the best value of $\mathrm{K}$. The results were drawn using pophelper R package 2.3.0 [14].

\section{3-population test and $\mathrm{F} 4$ ratio test}

The 3-population test and $\mathrm{f} 4$ ratio test were conducted on the dataset which contained Aboriginal Taiwanese using the AdmixTools package version 4.1 [43]. As an outgroup for the $\mathrm{f} 4$ ratio test, the HapMap data of 60 unrelated individuals from Yoruba in Ibadan, Nigeria (YRI) [61] were also merged. After merging all the datasets, those SNPs with a genotyping rate lower than 0.95 were filtered out, leaving 49,523 SNPs to be used.

The $\mathrm{f} 3(\mathrm{C} ; \mathrm{A}, \mathrm{B})$ statistics should be negative if a population $C$ has descended from a mixture of populations $\mathrm{A}$ and B. Assuming $\mathrm{CHB}$ or Taiwanese as a proxy of Asian-related and Gidra as that of Papuan-related ancestors, f3(Tonga; CHB or Taiwanese, Gidra) was calculated to test if admixture occurred in the ancestors of Tonga population.

An f4 ratio test, assuming the population relationships shown in Additional file 1: Fig. S2, was performed to estimate the proportion of admixture. The proportion of Asian-related and Papuan-related ancestry, $\alpha$ and 1- $\alpha$, respectively, was estimated by computing the ratio of two f4 statistics:

$\alpha=\mathrm{f} 4(\mathrm{CHB}, \mathrm{YRI}$; Tonga, Gidra)/f4(CHB, YRI; Taiwan, Gidra) $[43,51]$.

\section{Genome-wide scan for natural selection}

To detect signals of natural selection having acted over the genomic regions derived from Papuan-related ancestors in Polynesian genomes, ELAI analysis [15] was preformed across the genome for 23 Tongan subjects assuming $\mathrm{CHB}$ and Gidra as a proxy for their Asianand Papuan- related ancestors, respectively. The ELAI method computes expected ancestry dosage at each marker for each individual using a two-layer hidden Markov Model. To exclude close relatives, the IdenticalBy-Descent (IBD) value of each pair of individuals was checked. The calculation of IBD values was performed after LD pruning using PLINK software v1.90b5.2 [10] with the following settings, which define window size, step and the $r^{2}$ threshold: -indep-pairwise 5050.5 . Since one pair of individuals showed an IBD value higher than 0.125 (IBD value $=0.5185)$, one individual from this pair was excluded from the following analyses.

A total of 162,358 autosomal SNPs that showed a genotyping rate higher than 0.95 and that were polymorphic in each population were used for ELAI analysis. ELAI analysis was performed with the ELAI version 1.00 software with settings which defined the number of EM steps as 20, the upper layer number of clusters as 2, and lower layer number of clusters as 10 , in accordance with 
the manual [15]. Since the ancestors of Polynesians were considered to have reached Oceania about 3000 years ago, the admixture generations were set as 100 , which is consistent with the dates of admixture for Polynesian populations estimated in the previous studies: 83 (95\% CI 66-112) generations [46], 90 generations (95\% CI 77-131) [47], and 99 generations (95\% CI 19-267) [66]. Statistical analysis was conducted using $\mathrm{R}$ version 3.5 .3 (https://www.R-project.org/). The mean value of Papuanrelated ancestry proportion at each SNP among Tongan subjects was calculated and plotted across the genome using R package "ggplot2" version 3.1.1 [64]. The list of NCBI RefSeq genes in the detected regions was downloaded from the UCSC Table Browser and implemented in the UCSC Genome Browser [23, 25].

The ELAI analysis was also conducted assuming Aboriginal Taiwanese (Ami and Atayal, $\mathrm{n}=35$ ) [30, 46, 48] as a proxy for Asian-related ancestors. The analysis was performed on the dataset consisting of 49,523 autosomal SNPs with the same parameters as above. In addition, to examine the effect of recent European contact, the ELAI analysis was conducted with three-way admixed model assuming $\mathrm{CHB}$, Gidra and CEU $(\mathrm{n}=40)$ as a proxy for Asian-, Papuan- and European- related ancestry, respectively. The analysis was performed on the dataset consisting of 198,803 autosomal SNPs with the same parameters as above.

\section{Coalescent-based simulation}

Coalescent simulations under the assumption of selective neutrality were performed to address whether genetic drift alone could produce similar patterns of admixture across the genome to the ones observed in Tongan subjects. Coalescent-based simulations were performed using the R package "scrm" version 1.7.3.1 [58]. To reproduce the population history of Gidra, CHB and Tonga, a simple population history was assumed based on a single dispersal model into Asia [33, 35, 41] was assumed as described below. First, two subpopulations (Anc1 and Anc2) diverged from one ancestral population 1667 generations ago, which corresponds to 50,000 years ago when generation time is 30 years. Next, subpopulations diverged from Anc1 and Anc2, respectively, and admixed with each other 100 generations ago (3000 years ago). The descendants of Anc1 and Anc2 were regarded as Gidra and $\mathrm{CHB}$, respectively, and the admixed population was regarded as the Tongan (Polynesians) population. Segregating sites within a $1 \mathrm{Mb}$-long sequence were sampled 3000 times (i.e., 3 Gb long) for 48,46 and 90 chromosomes from hypothetical Gidra, Tongan and $\mathrm{CHB}$ populations, respectively. The mutation rate and recombination rate were set as $1.2 \times 10^{-8} /$ base/generation and $1.3 \times 10^{-8} /$ base/generation $[3,29]$, respectively.
The admixture rate in the simulation was given from the mean proportions of Papuan-related ancestry and Asian-related ancestry estimated in the ELAI analysis. The genotype data for 24, 23 and 45 individuals from the hypothetical Gidra, Tongan and $\mathrm{CHB}$ populations were generated from the sequences obtained. Considering the SNP ascertainment bias observed in real data, 162,358 SNPs, which exhibited the similar distribution of minor allele frequencies in the real data, were randomly extracted from the simulated genotype data. The above coalescent simulations were performed several times for various population sizes $(\mathrm{N}=250,500,750,1,000,5,000$ and 10,000 for each population). Since the mean and SD of Papuan-related ancestry estimated from simulation data for $\mathrm{N}=1,000$ were most similar to those of real data (Mean $=24.0 \%, \mathrm{SD}=10.3 \%$ ), we therefore set a population size of 1,000 for each population in the following analysis. The $\mathrm{R}$ code for coalescent simulation under the assumption of selective neutrality is provided in Additional file 2.

To evaluate the family-wise error rate (FWER) and false discovery rate (FDR) of our approach, we performed 100 independent runs of coalescent-based simulations and subsequent ELAI analyses with the same settings as described above. The number of independent genomic regions that exceeded $5 \mathrm{SD}$ from the mean was counted for each simulation run. Here, the mean and SD of Papuan-related ancestry were determined in each run.

\section{Linkage disequilibrium analysis}

To identify a tag SNP for rs17822931-C derived from Papuan-related ancestors, the LD $\left(D^{\prime}\right.$ and $\left.r^{2}\right)$ of rs17822931 with other SNPs in the franking region of the $A B C C 11$ was evaluated in 14 Papuans from Simons Genome Diversity Project [33] using Haploview 4.1 [5]. Then, an SNP, rs12445647, which was in strong LD with rs17822931 in SGDP Papuans $\left(D^{\prime}=1\right.$ and $\left.r^{2}=1\right)$ and observed in high frequency in Papuans but low frequency in populations from the 1000 Genomes Project Phase 3 [1], was selected as a tag SNP. The LD of rs17822931 with rs12445647 was evaluated in each population of YRI, CEU, CHB, JPT, CHS, CDX, and KHV in the 1000 Genomes Project Phase 3 (1000 Genomes Project Consortium et al. 2015) using LDlink [32].

\section{Genotyping rs 17822931 and rs12445647 in Oceanian populations}

Two SNPs, rs17822931 and rs12445647, were genotyped by the TaqMan assay for a total of 616 adult subjects (18 years old or older) from four Oceanian populations: Tongan $(n=174)$, Munda $(n=170)$, Gidra $(n=165)$ and Rawaki $(\mathrm{n}=107)$. Munda people were AN-speaking Melanesians in the New Georgia Islands in the western part 
of the Solomon Islands. Rawaki village was also located in the Solomon Islands but the inhabitants were regarded as AN-speaking Micronesians as they had migrated there from the overpopulated Gilbert Islands (Kiribati) in the 1960s [40]. Blood sampling was conducted after obtaining informed consent from each subject. Genomic DNA was extracted from peripheral blood using a QIAamp Blood Kit (Qiagen, Hilden, Germany). The LD of rs17822931 with rs12445647 was evaluated in each Oceanian population using Haploview 4.1 [5].

\section{Approximate Bayesian computation for estimation of selection coefficient}

The approximate Bayesian computation was used to estimate the selection coefficient $(s)$ for rs17822931-C. We used a forward-time simulation assuming the relative fitness of the CC, CT, and TT genotypes at rs17822931 to be $1,1-s$, and 1-2 s, respectively. The change in allele frequency of rs17822931-C was modeled as follows: the expected allele frequency of rs17822931-C at generation $t+1$ is given by

$$
p_{t+1}=\frac{p_{t}^{2}+p_{t}\left(1-p_{t}\right)(1-s)}{p_{t}^{2}+2 p_{t}\left(1-p_{t}\right)(1-s)\left(1-p_{t}\right)^{2}(1-2 s)},
$$

where $p_{t}$ is the allele frequency of rs17822931-C in a Polynesian population at generation $t$ since the admixture of Papuan-related and Asian-related ancestors. Assuming that the population size, $N$, is constant, $p_{t}$ is expressed by $i_{t} / 2 \mathrm{~N}$, where $i_{t}$ is the number of copies of rs17822931-C at generation $t$. The number of copies of rs17822931-C at generation $t+1$ follows the binomial distribution:

$$
\operatorname{Pr} o b\left(i_{+1} \mid p_{t}\right)=\left(\begin{array}{c}
2 N \\
i_{t+1}
\end{array}\right) p_{t+1}^{i_{t}+1}\left(1-p_{t+1}\right)^{2 N-i_{t+1}}
$$

In the computer simulation, $i_{t+1}$ is generated as a random number based on $P_{t}$. The initial allele frequency of rs17822931-C in a Polynesian population soon after admixture was given based on the present allele frequencies in Papuan (Gidra) and Asian (CHB) populations (i.e. 0.915 and 0.049 , respectively) and the admixture proportion estimated by ELAI analysis (i.e. 0.246 for Papuanrelated ancestry and 0.754 for Asian-related ancestry) as follows: $0.915 * 0.246+0.049 * 0.754=0.26$. The population size, $N$, was set as 1,000 . In each simulation run, the value of $s$ was randomly generated using a uniform distribution in the range $(0,1)$. The value of $s$ was recorded only when the allele frequency of rs17822931-C after 100 generations, corresponding to 3000 years, fell within $\pm 5 \%$ of the observed allele frequency in the present Tongan population (i.e. 0.722 to 0.798 ). The mean and $95 \%$ credible interval of $s$ were calculated for 10,000 successful runs. The computer simulation mentioned above was implemented in $\mathrm{R}$ 3.5.3. The $\mathrm{R}$ code for approximate Bayesian computation with forward simulation is provided in Additional file 3.

\section{Comparison of the expression level of $A B C C 11$ between haplotypes}

The expression level of $A B C C 11$ was compared between haplotypes harboring rs12445647-T and rs17822931$\mathrm{C}$ and harboring rs12445647-T and rs17822931-C using publicly available data: genotype data of the 1000 Genomes Project Phase 3 populations [1] and microarray data of the HapMap3 populations $[13,18,59]$ obtained from the ArrayExpress database at EMBL-EBI (www.ebi.ac.uk/arrayexpress) under accession number E-MTAB-264. A total of 217 unrelated subjects with the rs17822931-CC genotype commonly included in the two datasets were used for a regression analysis, where the independent variable of rs12445647 was coded as the number of copies of rs12445647-T (i.e., $\mathrm{GG}=0, \mathrm{GT}=1$, $\mathrm{TT}=2$ ). The 217 subjects belonged to $\mathrm{CHB}, \mathrm{GIH}, \mathrm{JPT}$, LWK, MEX, MKK and YRI in the 1000 Genomes Project.

\begin{abstract}
Abbreviations
SD: Standard deviation; $\mathrm{XMHC}$ : Extended major histocompatibility complex; ABCC11: ATP-binding cassette transporter sub-family C member 11; AN: Austronesian; HLA: Human leukocyte antigen; PCA: Principal component analysis; CHB: Han Chinese population of Beijing; ELAl: Effective Local Ancestry Inference; FDR: False discovery rate (FDR); FWER: Family-wise error rate; LD: Linkage disequilibrium.
\end{abstract}

\section{Supplementary Information}

The online version contains supplementary material available at https://doi. org/10.1186/s12862-021-01900-y.

Additional file 1. Additional figures.

Additional file 2. $R$ code for coalescent simulation under the assumption of selective neutrality.

Additional file 3. $\mathrm{R}$ code for approximate Bayesian computation with forward simulation.

\section{Acknowledgements}

We are deeply grateful to people from the Kingdom of Tonga for their kind cooperation in providing blood samples for genotyping. We thank Drs. Taniela Palu (Ministry of Health, Kingdom of Tonga), Viliami Tangi (Diabetes Clinic, Kingdom of Tonga), and Kazumichi Katayama (Primate Research Institute, Kyoto University) for research on the Tongan population. We also wish to acknowledge Dr. Irina Pugach and Prof. Mark Stoneking, who kindly provided the genotype data of Taiwanese populations. This study was partly supported by a Grant-in-Aid for Scientific Research from the Ministry of Education, Culture, Sports, Science, and Technology of Japan.

\section{Authors' contributions}

MI conceived the study. MI designed the analyses. RK, TF, KN, TY, MN, TI, RE, TI, YM, RO, and JO collected the samples. RK and I.N. extracted DNA from blood samples. MI conducted the SNP genotyping experiment. MI carried out the statistical analyses and computer simulations. MI wrote the manuscript with support from JO. JO supervised the project. All authors read and approved the final manuscript. 


\section{Funding}

This work was partly supported by JSPS KAKENHI Grant Number 25291103 , JSPS KAKENHI Grant Number 21H02570, and Grant-in-Aid for JSPS Fellows Grant Number 19J12435. The funding bodies played no role in the design of the study and collection, analysis, and interpretation of data and in writing the manuscript.

\section{Availability of data and materials}

The data newly created in this study are available on request from the corresponding author. The data are not publicly available due to privacy or ethical restrictions.

\section{Declarations}

\section{Ethics approval and consent to participate}

This study was approved by the National Health Ethics \& Research Committee of Tonga, and the Research Ethics Committees of The University of Tokyo and of the Faculty of Medicine, The University of Tokyo. A written informed consent was obtained from each participant.

\section{Consent for publication}

Not applicable.

\section{Competing interests}

The authors declare no competing interests.

\section{Author details}

'Department of Biological Sciences, Graduate School of Science, The University of Tokyo, Tokyo 113-0033, Japan. ${ }^{2}$ Department of Human Biology and Anatomy, Graduate School of Medicine, University of the Ryukyus, Nishihara 903-0125, Japan. ${ }^{3}$ Genome Medical Science Project, Research Center for Hepatitis and Immunology, National Center for Global Health and Medicine, Chiba 272-8516, Japan. ${ }^{4}$ Graduate School of Asian and African Area Studies, Kyoto University, Kyoto 606-8501, Japan. ${ }^{5}$ Department of International Health and Nursing, Faculty of Nursing, Toho University, Tokyo 143-0015, Japan. ${ }^{6}$ Faculty of Health Sciences, Hokkaido University, Sapporo 060-0812, Japan. ${ }^{7}$ Graduate School of Health Sciences, Kobe University, Kobe 654-0142, Japan. ${ }^{8}$ Department of Human Ecology, Faculty of Agriculture, Saga University, Saga 840-8502, Japan. ${ }^{9}$ Faculty of Health and Nutrition, Bunkyo University, Chigasaki 253-8550, Japan. ${ }^{10}$ Japan Wildlife Research Center, Tokyo 130-8606, Japan

Received: 12 January 2021 Accepted: 25 August 2021

Published online: 22 September 2021

\section{References}

1. 1000 Genomes Project Consortium, Auton A, Brooks LD, Durbin RM, Garrison EP, Kang HM, Korbel JO, Marchini JL, McCarthy S, McVean GA, et al. A global reference for human genetic variation. Nature. 2015;526:68-74 https://doi.org/10.1038/nature15393.

2. Alexander DH, Novembre J, Lange K. Fast model-based estimation of ancestry in unrelated individuals. Genome Res. 2009;19:1655-64. https:// doi.org/10.1101/gr.094052.109.

3. Altshuler DL, Durbin RM, Abecasis GR, Bentley DR, Chakravarti A, Clark AG, Collins FS, De La Vega FM, Donnelly P, Egholm M, et al. A map of human genome variation from population-scale sequencing. Nature. 2010;467:1061-73. https://doi.org/10.1038/nature09534.

4. Athens JS, Toggle HD, Ward JV, Welch DJ. Avifaunal extinctions, vegetation change, and Polynesian impacts in prehistoric Hawai'i. Archaeo Ocean. 2002;37:57-78. https://doi.org/10.1002/j.1834-4453.2002.tb005 07.x.

5. Barrett JC, Fry B, Maller J, Daly MJ. Haploview: analysis and visualization of LD and haplotype maps. Bioinformatics. 2005. https://doi.org/10.1093/ bioinformatics/bth457.

6. Bellwood P, Burns E. The Batanes archaeological project and the" outof-Taiwan" hypothesis for Austronesian dispersal. J Austronesian Stud. 2005;1:1-36.
7. Bhatia G, Tandon A, Patterson N, Aldrich MC, Ambrosone CB, Amos C, Bandera EV, Berndt SI, Bernstein L, Blot WJ, et al. Genome-wide scan of 29,141 African Americans finds no evidence of directional selection since admixture. Am J Hum Genet. 2014;95:437-44. https://doi.org/10.1016/j. ajhg.2014.08.011.

8. Brisbin A, Bryc K, Byrnes J, Zakharia F, Omberg L, Degenhardt J, Reynolds A, Ostrer H, Mezey JG, Bustamante CD. PCAdmix: principal componentsbased assignment of ancestry along each chromosome in individuals with admixed ancestry from two or more populations. Hum Biol. 2012;84:343-64. https://doi.org/10.3378/027.084.0401.

9. Burley DV, Dickinson WR, Barton A, Shutler R. Lapita on the Periphery. New data on old problems in the Kingdom of Tonga. Archaeol Ocean. 2001;36:89-104. https://doi.org/10.1002/j.1834-4453.2001.tb00481.x.

10. Chang CC, Chow CC, Tellier LCAM, Vattikuti S, Purcell SM, Lee JJ. Secondgeneration PLINK: rising to the challenge of larger and richer datasets. Gigascience. 2015;4:7. https://doi.org/10.1186/s13742-015-0047-8.

11. Chen ZS, Guo Y, Belinsky MG, Kotova E, Kruh GD. Transport of bile acids, sulfated steroids, estradiol 17- $\beta$-D- glucuronide, and leukotriene C4 by human multidrug resistance protein 8 (ABCC 11). Mol Pharmacol. 2005:67:545-57. https://doi.org/10.1124/mol.104.007138.

12. Deng L, Ruiz-Linares A, Xu S, Wang S. Ancestry variation and footprints of natural selection along the genome in Latin American populations. Sci Rep. 2016;6:1-7. https://doi.org/10.1038/srep21766.

13. Dimas AS, Nica AC, Montgomery SB, Stranger BE, Raj T, Buil A, Giger T, Lappalainen T, Gutierrez-Arcelus M, McCarthy MI, et al. Sex-biased genetic effects on gene regulation in humans. Genome Res. 2012. https://doi. org/10.1101/gr.134981.111.

14. Francis RM. POPHELPER: an R package and web app to analyse and visualise population structure. Mol Ecol Resour. 2017;17:27-32. https:// doi.org/10.1111/1755-0998.12509.

15. Guan Y. Detecting structure of haplotypes and local ancestry. Genetics. 2014;196:625-42. https://doi.org/10.1534/genetics.113.160697.

16. Hellenthal G, Busby GBJ, Band G, Wilson JF, Capelli C, Falush D, Myers S. A genetic atlas of human admixture history. Science. 2014;343:747-51. https://doi.org/10.1126/science.1243518.

17. Hogg AG, Higham TFG, Lowe DJ, Palmer JG, Reimer PJ, Newnham RM. A wiggle-match date for Polynesian settlement of New Zealand. Antiquity. 2003:77:116-25. https://doi.org/10.1017/S0003598X00061408.

18. Houldcroft CJ, Petrova V, Liu JZ, Frampton D, Anderson CA, Gall A, Kellam P. Host genetic variants and gene expression patterns associated with epstein-barr virus copy number in lymphoblastoid cell lines. PLoS ONE. 2014. https://doi.org/10.1371/journal.pone.0108384.

19. Hunt TL, Lipo CP. Late colonization of Easter Island. Science. 2006;311:1603-6. https://doi.org/10.1126/science.1121879.

20. Ioannidis AG, Blanco-Portillo J, Sandoval K, Hagelberg E, Miquel-Poblete JF, Moreno-Mayar JV, Rodríguez-Rodríguez JE, Quinto-Cortés CD, Auckland K, Parks T, et al. Native American gene flow into Polynesia predating Easter Island settlement. Nature. 2020. https://doi.org/10.1038/ s41586-020-2487-2.

21. Isshiki M, Naka I, Watanabe Y, Nishida N, Kimura R, Furusawa T, Natsuhara K, Yamauchi T, Nakazawa M, Ishida T, et al. Admixture and natural selection shaped genomes of an Austronesian-speaking population in the Solomon Islands. Sci Rep. 2020;10:6872. https://doi.org/10.1038/ s41598-020-62866-3.

22. Johnson NA, Coram MA, Shriver MD, Romieu I, Barsh GS, London SJ, Tang $H$. Ancestral Components of Admixed Genomes in a Mexican Cohort. Copenhaver GP, ed. PLoS Genet. 2011; 7:e1002410. https://doi.org/10. 1371/journal.pgen.1002410.

23. Karolchik D. The UCSC table browser data retrieval tool. Nucleic Acids Res 2004;32:493D - 496. https://doi.org/10.1093/nar/gkh103.

24. Kayser M, Lao O, Saar K, Brauer S, Wang X, Nürnberg P, Trent RJ, Stoneking M. Genome-wide analysis indicates more Asian than Melanesian Ancestry of Polynesians. Am J Hum Genet. 2008;82:194-8. https://doi.org/10. 1016/j.ajhg.2007.09.010

25. Kent JW, Sugnet CW, Furey TS, Roskin KM, Pringle TH, Zahler AM, Haussler D. The human genome browser at UCSC. Genome Res. 2002;12(9961006):2002. https://doi.org/10.1101/gr.229102.

26. Kimura R, Fujimoto A, Tokunaga K, Ohashi J. 2007. A Practical Genome Scan for Population-Specific Strong Selective Sweeps That Have Reached 
Fixation. Harpending H, ed. PLoS One 2:e286. https://doi.org/10.1371/ journal.pone.0000286.

27. Kimura R, Ohashi J, Matsumura Y, Nakazawa M, Inaoka T, Ohtsuka R, Osawa M, Tokunaga K. Gene flow and natural selection in oceanic human populations inferred from genome-wide SNP typing. Mol Biol Evol. 2008;25:1750-61. https://doi.org/10.1093/molbev/msn128.

28. Kirch PV, Hunt TL. Radiocarbon dates from the Mussau Islands and the Lapita Colonization of the Southwestern Pacific. Radiocarbon. 1988:30:161-9. https://doi.org/10.1017/S0033822200044106.

29. Kong A, Frigge ML, Masson G, Besenbacher S, Sulem P, Magnusson G, Gudjonsson SA, Sigurdsson A, Aslaug J, Adalbjorg J, et al. Rate of de novo mutations and the importance of father's age to disease risk. Nature. 2012:488:471-5. https://doi.org/10.1038/nature11396.

30. Lazaridis I, Patterson N, Mittnik A, Renaud G, Mallick S, Kirsanow K Sudmant PH, Schraiber JG, Castellano S, Lipson M, et al. Ancient human genomes suggest three ancestral populations for present-day Europeans. Nature. 2014;513:409-13. https://doi.org/10.1038/nature13673.

31. Lipson M, Skoglund P, Spriggs M, Valentin F, Bedford S, Shing R, Buckley H, Phillip I, Ward GK, Mallick S, et al. Population turnover in remote oceania shortly after initial settlement. Curr Biol. 2018;28:1157-1165.e7. https:// doi.org/10.1016/j.cub.2018.02.051.

32. Machiela MJ, Chanock SJ. LDlink: A web-based application for exploring population-specific haplotype structure and linking correlated alleles of possible functional variants. Bioinformatics. 2015;31:3555-7. https://doi. org/10.1093/bioinformatics/btv402.

33. Mallick S, Li H, Lipson M, Mathieson I, Gymrek M, Racimo F, Zhao M, Chennagiri $\mathrm{N}$, Nordenfelt $\mathrm{S}$, Tandon $\mathrm{A}$, et al. The simons genome diversity project: 300 genomes from 142 diverse populations. Nature. 2016;538:201-6. https://doi.org/10.1038/nature18964.

34. Martin A, Saathoff M, Kuhn F, Max H, Terstegen L, Natsch A. A functional ABCC11 allele is essential in the biochemical formation of human axillary odor. J Invest Dermatol. 2010;130:529-40. https://doi.org/10.1038/jid. 2009.254.

35. Mellars P, Gori KC, Carr M, Soares PA, Richards MB. Genetic and archaeological perspectives on the initial modern human colonization of southern Asia. Proc Natl Acad Sci U S A. 2013;1 10:10699-704. https://doi.org/ 10.1073/pnas.1306043110.

36. Miura K, Yoshiura K, Miura S, Shimada T, Yamasaki K, Yoshida A, Nakayama D, Shibata Y, Niikawa N, Masuzaki H. A strong association between human earwax-type and apocrine colostrum secretion from the mammary gland. Hum Genet. 2007;121:631-3. https://doi.org/10.1007/ s00439-007-0356-9.

37. Norris ET, Rishishwar L, Chande AT, Conley AB, Ye K, Valderrama-Aguirre A, Jordan IK. Admixture-enabled selection for rapid adaptive evolution in the Americas. Genome Biol. 2020;21:29. https://doi.org/10.1186/ s13059-020-1946-2.

38. O'Connell JF, Allen J. The process, biotic impact, and global implications of the human colonization of Sahul about 47,000 years ago. J Archaeol Sci. 2015;56:73-84. https://doi.org/10.1016/j.jas.2015.02.020

39. Ohashi J, Naka I, Tsuchiya N. The impact of natural selection on an ABCC11 SNP determining earwax type. Mol Biol Evol. 2011;28:849-57. https://doi.org/10.1093/molbev/msq264.

40. Ohashi J, Naka I, Kimura R, Tokunaga K, Yamauchi T, Natsuhara K, Furusawa T, Yamamoto R, Nakazawa M, Ishida T, et al. Polymorphisms in the $\mathrm{ABO}$ blood group gene in three populations in the New Georgia group of the Solomon Islands. J Hum Genet. 2006;51:407-11. https://doi.org/10. 1007/s10038-006-0375-8.

41. Oppenheimer S. Out-of-Africa, the peopling of continents and islands: tracing uniparental gene trees across the map. Philos Trans R Soc B Biol Sci. 2012;367:770-84. https://doi.org/10.1098/rstb.2011.0306.

42. Patin E, Lopez M, Grollemund R, Verdu P, Harmant C, Quach H, Laval G, Perry GH, Barreiro LB, Froment A, et al. Dispersals and genetic adaptation of Bantu-speaking populations in Africa and North America. Science. 2017:356:543-6. https://doi.org/10.1126/science.aal1988.

43. Patterson N, Moorjani P, Luo Y, Mallick S, Rohland N, Zhan Y, Genschoreck T, Webster T, Reich D. Ancient admixture in human history. Genetics. 2012;192:1065-93. https://doi.org/10.1534/genetics.112.145037.

44. Pierron D, Heiske M, Razafindrazaka H, Pereda-Loth V, Sanchez J, Alva O, Arachiche A, Boland A, Olaso R, Deleuze JF, et al. Strong selection during the last millennium for African ancestry in the admixed population of Madagascar. Nat Commun. 2018;9:1-9. https://doi.org/10.1038/ s41467-018-03342-5.

45. Pritchard JK, Stephens M, Donnelly P. Inference of population structure using multilocus genotype data. Genetics. 2000;155:945-59.

46. Pugach I, Duggan AT, Merriwether DA, Friedlaender FR, Friedlaender JS, Stoneking M. The gateway from near into remote oceania: new insights from genome-wide data. Mol Biol Evol. 2018;35:871-86. https://doi.org/ 10.1093/molbev/msx333.

47. Pugach I, Matveyev R, Wollstein A, Kayser M, Stoneking M. Dating the age of admixture via wavelet transform analysis of genome-wide data. Genome Biol. 2011;12:R19. https://doi.org/10.1186/gb-2011-12-2-r19.

48. Qin P, Stoneking M. Denisovan ancestry in East Eurasian and Native American populations. Mol Biol Evol. 2015;32:2665-74. https://doi.org/10. 1093/molbev/msv141.

49. Quintana-Murci L. Human immunology through the lens of evolutionary genetics. Cell. 2019;177:184-99. https://doi.org/10.1016/i.cell.2019.02.033.

50. Racimo F, Sankararaman S, Nielsen R, Huerta-Sánchez E. Evidence for archaic adaptive introgression in humans. Nat Rev Genet. 2015;16:35971. https://doi.org/10.1038/nrg3936.

51. Reich $D$, Thangaraj K, Patterson N, Price AL, Singh L. Reconstructing Indian population history. Nature. 2009;461:489-94. https://doi.org/10.1038/ nature08365.

52. Rieth T, Cochrane EE. 2015. The chronology of colonization in Remote Oceania. In: Cochrane EE, Hunt TL, eds. Oxford University Press.

53. Rieth $T M$, Hunt TL. A radiocarbon chronology for Sāmoan prehistory. J Archaeol Sci. 2008;35:1901-27. https://doi.org/10.1016/j.jas.2007.12.001.

54. Rishishwar L, Conley AB, Wigington $\mathrm{CH}$, Wang L, Valderrama-Aguirre $\mathrm{A}$, King Jl. Ancestry, admixture and fitness in Colombian genomes. Sci Rep. 2015:5:12376. https://doi.org/10.1038/srep12376.

55. Sabeti PC, Reich DE, Higgins JM, Levine HZP, Richter DJ, Schaffner SF, Gabriel SB, Platko JV, Patterson NJ, McDonald GJ, et al. Detecting recent positive selection in the human genome from haplotype structure. Nature. 2002;419:832-7. https://doi.org/10.1038/nature01140.

56. Skoglund P, Posth C, Sirak K, Spriggs M, Valentin F, Bedford S, Clark GR, Reepmeyer C, Petchey F, Fernandes D, et al. Genomic insights into the peopling of the Southwest Pacific. Nature. 2016;538:510-3. https://doi. org/10.1038/nature19844.

57. Spriggs M. Chronology of the neolithic transition in Island Southeast Asia and the Western Pacific: a view from 2003. Rev Archaeol. 2003;24:57-80.

58. Staab PR, Zhu S, Metzler D, Lunter G. Scrm: Efficiently simulating long sequences using the approximated coalescent with recombination. Bioinformatics. 2015:31:1680-2. https://doi.org/10.1093/bioinformatics/ btu861.

59. Stranger BE, Montgomery SB, Dimas AS, Parts L, Stegle O, Ingle CE, Sekowska M, Smith GD, Evans D, Gutierrez-Arcelus M, et al. 2012. Patterns of Cis Regulatory Variation in Diverse Human Populations. Barsh GS, ed. PLoS Genet. 8:e1002639. https://doi.org/10.1371/journal.pgen.1002639.

60. Tang H, Choudhry S, Mei R, Morgan M, Rodriguez-Cintron W, Burchard EG, Risch NJ. Recent genetic selection in the ancestral admixture of Puerto Ricans. Am J Hum Genet. 2007;81:626-33. https://doi.org/10.1086/ 520769.

61. The International HapMap Consortium. A haplotype map of the human genome. Nature. 2005:437:1299-320. https://doi.org/10.1038/natur e04226.

62. Uruakpa F, Ismond MA, Akobundu EN. Colostrum and its benefits: a review. Nutr Res. 2002;22:755-67. https://doi.org/10.1016/50271-5317(02) 00373-1.

63. Voight BF, Kudaravalli S, Wen X, Pritchard JK. 2006. A Map of Recent Positive Selection in the Human Genome. Hurst L, ed. PLoS Biol. 4:e72. https://doi.org/10.1371/journal.pbio.0040072.

64. Wickham H. ggplot2: Elegant Graphics for Data Analysis. New York, NY: Springer New York; 2016

65. Wickler S, Spriggs M. Pleistocene human occupation of the Solomon Islands, Melanesia. Antiquity. 1988;62:703-6. https://doi.org/10.1017/ S0003598X00075104.

66. Wollstein A, Lao O, Becker C, Brauer S, Trent RJ, Nürnberg P, Stoneking M, Kayser M. Demographic history of oceania inferred from genome-wide data. Curr Biol. 2010;20:1983-92. https://doi.org/10.1016/j.cub.2010.10. 040 . 
67. Yoshiura KI, Kinoshita A, Ishida T, Ninokata A, Ishikawa T, Kaname T, Bannai M, Tokunaga K, Sonoda S, Komaki R, et al. A SNP in the ABCC11 gene is the determinant of human earwax type. Nat Genet. 2006;38:324-30. https://doi.org/10.1038/ng1733.

68. Zhou Q, Zhao L, Guan Y. Strong selection at MHC in Mexicans since Admixture. PLoS Genet. 2016;12:1-17. https://doi.org/10.1371/journal. pgen.1005847.

\section{Publisher's Note}

Springer Nature remains neutral with regard to jurisdictional claims in published maps and institutional affiliations.
Ready to submit your research? Choose BMC and benefit from:

- fast, convenient online submission

- thorough peer review by experienced researchers in your field

- rapid publication on acceptance

- support for research data, including large and complex data types

- gold Open Access which fosters wider collaboration and increased citations

- maximum visibility for your research: over 100M website views per year

At BMC, research is always in progress.

Learn more biomedcentral.com/submissions 